\title{
Crystal structure of 2-(6-[2-(5-ethyl-5-hydroxy-6-methyl-tetrahydro-pyran- 2-yl)-15-oxo-2,10,12-trimethyl-1,6,8-trioxa-dispiro[4.1.5.3]pentadec-13-en- 9-yl]-2-hydroxy-1,3-dimethyl-4-oxo-heptyl-5-methyl-tetrahydro-pyran- 2-yl)-butyrate sodium, $\mathrm{Na}\left(\mathrm{C}_{42} \mathrm{H}_{67} \mathrm{O}_{11}\right)$, SY-9 - antibiotic 20-oxo-salinomycin
}

\author{
E. F. Paulus*,I and L. Vértesy ${ }^{\mathrm{II}}$ \\ I Johann Wolfgang Goethe-Universität, Institut für Mineralogie/Kristallographie, Senckenberganlage 30, D-60325 Frankfurt/Main, Germany \\ II Aventis Pharma Deutschland GmbH, Natural Product Research, D-65926 Frankfurt/Main, Germany
}
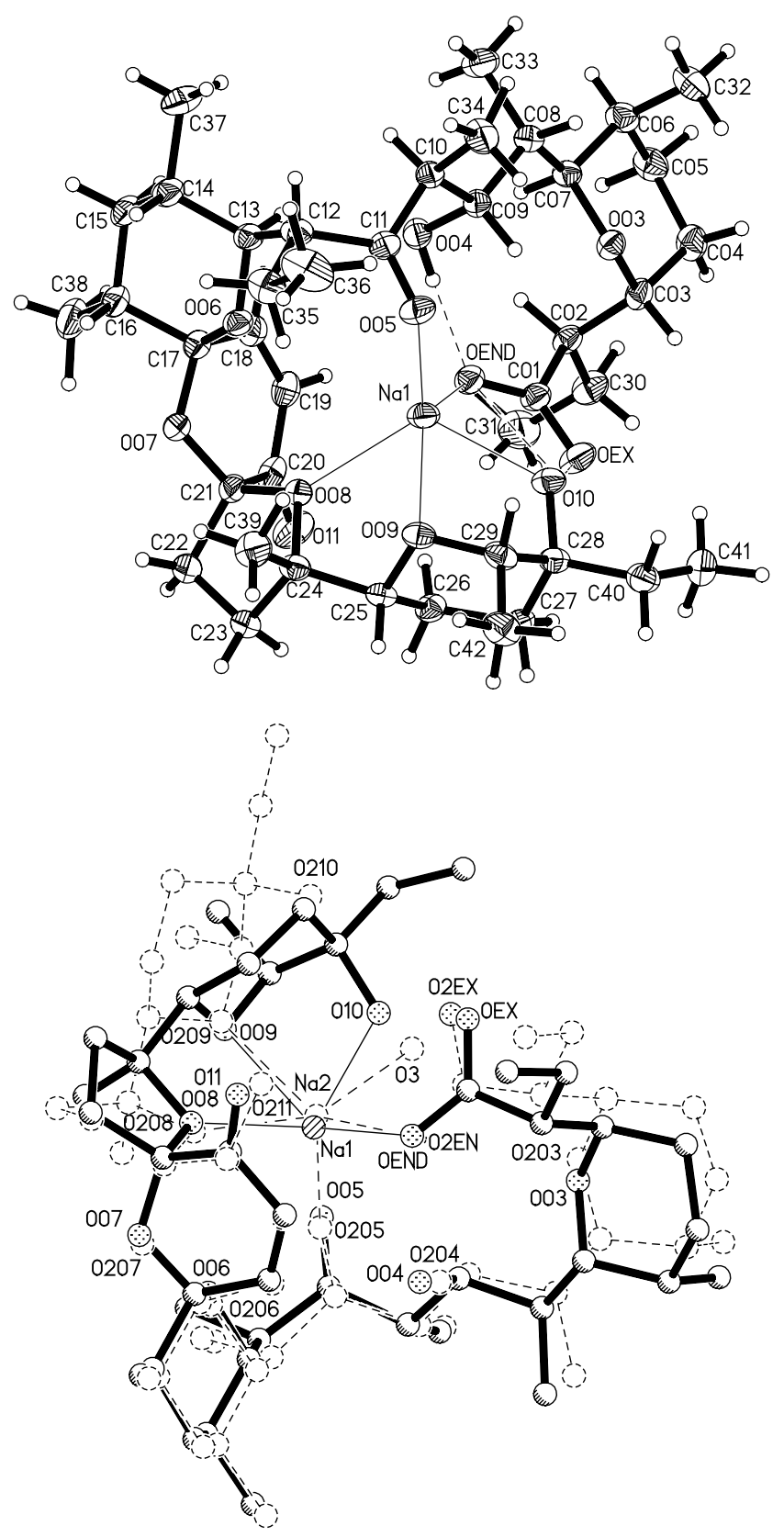

\begin{abstract}
$\mathrm{C}_{42} \mathrm{H}_{67} \mathrm{NaO}_{11}$, orthorhombic, $P 2{ }_{1} 2{ }_{1} 2_{1}$ (no. 19), $a=10.5617(5) \AA$, $b=19.1580(9) \AA, c=21.5366(9) \AA, V=4357.7 \AA^{3}, Z=4$, $R_{\mathrm{gt}}(F)=0.035, w R_{\mathrm{ref}}\left(F^{2}\right)=0.082, T=293 \mathrm{~K}$.
\end{abstract}

\section{Source of material}

The title compound, the antibiotic SY-9 [1] is produced by fermentation with Streptomyces albus ATCC 21838 as a minor component, while salinomycin is the main product. Although it is a salt, crystals were grown from $n$-hexane.

\section{Experimental details}

The average $I / \sigma$ of the measured X-ray intensities is 37.9. All hydrogens could be determined experimentally. The largest difference peak and hole were 0.098 and $-0.117 \mathrm{e} / \AA^{3}$, respectively. The Flack parameter is -0.2(4) [2]. According to Hamilton [3] the hypothesis, that the given absolute configuration might be wrong, can be rejected at the $0.5 \%$ level $\left[\left(R_{1} / R_{2}\right)_{\exp }=0.0818 / 0.0817=\right.$

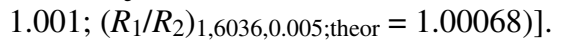

\section{Discussion}

SY-9 [1] is a member of the polyether family of antibiotics and is related to salinomycin [4]. The absolute configuration is $(2 R, 3 R$, $6 S, 7 R, 8 S, 9 S, 10 S, 12 R, 13 S, 14 S, 16 R, 17 R, 21 S, 24 S, 25 R, 28 R, 29 S)$, as it was analogously determined for salinomycin [5-8] and SY-1 [9]. In contrast to SY-1 there is no solvent molecule methanol as a remainder of the chromatographical separation from the ingredients of the original natural extract. Like in SY-1 and in salinomycin itself sodium has coordination number 5 (figure, top). Because there are no solvent water molecules like in salinomycin, $\mathrm{O} 10$ is taken for completing the coordination of sodium instead of $\mathrm{O} 02$ as in SY-1. Figure, bottom shows the least squares fit to molecule 2 (M2) of salinomycin, a molecule with a relatively large head-to-tail distance $d\left(\mathrm{O} 10 \mathrm{H} \cdots \mathrm{O}_{\text {exo positioned }}\right)=3.255 \AA$, fitting only sodium and three oxygens of its configuration. The fit to molecule 1 (M1) is much worse, because only in $\mathrm{M} 2 \mathrm{O}_{\text {endo positioned }}$ is coordinated to Na like in SY-9. $\mathrm{O}_{\text {endo positioned }}$ and $\mathrm{O}_{\text {exo positioned }}$ are the two oxygens at $\mathrm{C} 01$. The average e.s.d. for a $\mathrm{C}-\mathrm{C}$ bond length is $0.004 \AA$, for a $\mathrm{C}-\mathrm{O}$ bond length $0.003 \AA$, for a $\mathrm{C}-\mathrm{H}$ and $\mathrm{O}-\mathrm{H}$ bond length $0.03 \AA$, for $\mathrm{a} \mathrm{O}-\mathrm{Na}$ coordination distance $0.002 \AA$, for bond angles (without hydrogens) $0.2^{\circ}$ and for torsion angles $0.3^{\circ}$.

\footnotetext{
* Correspondence author (e-mail: e.paulus@kristall.uni-frankfurt.de)
} 
Table 1. Data collection and handling.

Crystal:

Wavelength:

$\mu$ :

Diffractometer, scan mode:

$2 \theta_{\text {max }}$ :

$N(h k l)_{\text {measured, }} N(h k l)_{\text {unique }}$

Criterion for $I_{\mathrm{obs}}, N(h k l)_{\mathrm{gt}}$ :

$N(\text { param })_{\text {refined: }}$

Programs:

colorless thin plate, size $0.1 \times 0.4 \times 0.5 \mathrm{~mm}$

Mo $K_{\alpha}$ radiation $(0.71073 \AA)$

$0.92 \mathrm{~cm}^{-1}$

Three-circle AXS/CCD, $\omega$ with $\Delta \omega=0.3^{\circ}$

$46.52^{\circ}$

22386, 6211

$I_{\mathrm{obs}}>2 \sigma\left(I_{\mathrm{obs}}\right), 5231$

755

SHELXS-97 [10], SHELXL-97 [11]

SHELXTL-PLUS [12]

Table 2. Atomic coordinates and displacement parameters (in $\AA^{2}$ ).

\begin{tabular}{|c|c|c|c|c|c|}
\hline Atom & Site & $x$ & $y$ & $z$ & $U_{\text {iso }}$ \\
\hline $\mathrm{H}(02)$ & $4 a$ & $0.755(2)$ & $0.793(1)$ & $0.902(1)$ & $0.045(6)$ \\
\hline $\mathrm{H}(03)$ & $4 a$ & $0.498(3)$ & $0.739(1)$ & $0.883(1)$ & $0.072(8)$ \\
\hline $\mathrm{H}(041)$ & $4 a$ & $0.643(3)$ & $0.638(2)$ & $0.885(1)$ & $0.09(1)$ \\
\hline $\mathrm{H}(042)$ & $4 a$ & $0.529(3)$ & $0.639(2)$ & $0.938(1)$ & $0.09(1)$ \\
\hline $\mathrm{H}(051)$ & $4 a$ & $0.791(3)$ & $0.676(1)$ & $0.956(1)$ & $0.068(8)$ \\
\hline $\mathrm{H}(052)$ & $4 a$ & $0.718(3)$ & $0.603(2)$ & $0.985(1)$ & $0.09(1)$ \\
\hline $\mathrm{H}(06)$ & $4 a$ & $0.728(3)$ & $0.695(2)$ & $1.063(1)$ & $0.068(9)$ \\
\hline $\mathrm{H}(07)$ & $4 a$ & $0.725(2)$ & $0.789(1)$ & $0.995(1)$ & $0.044(6)$ \\
\hline $\mathrm{H}(08)$ & $4 a$ & $0.517(2)$ & $0.804(1)$ & $1.083(1)$ & $0.055(7)$ \\
\hline $\mathrm{H}(09)$ & $4 a$ & $0.509(2)$ & $0.890(1)$ & $1.006(1)$ & $0.049(7)$ \\
\hline $\mathrm{H}(10)$ & $4 a$ & $0.628(3)$ & $0.957(1)$ & $1.110(1)$ & $0.065(8)$ \\
\hline $\mathrm{H}(12)$ & $4 a$ & $0.592(2)$ & $1.078(1)$ & $1.118(1)$ & $0.053(7)$ \\
\hline $\mathrm{H}(16)$ & $4 a$ & $0.912(3)$ & $1.193(2)$ & $0.996(1)$ & $0.068(8)$ \\
\hline $\mathrm{H}(14)$ & $4 a$ & $0.793(3)$ & $1.171(1)$ & $1.094(1)$ & $0.061(7)$ \\
\hline $\mathrm{H}(151)$ & $4 a$ & $1.016(3)$ & $1.145(2)$ & $1.084(1)$ & $0.076(9)$ \\
\hline $\mathrm{H}(152)$ & $4 a$ & $0.982(3)$ & $1.066(2)$ & $1.063(1)$ & $0.09(1)$ \\
\hline $\mathrm{H}(18)$ & $4 a$ & $0.903(3)$ & $1.003(2)$ & $0.976(1)$ & $0.075(9)$ \\
\hline $\mathrm{H}(19)$ & $4 a$ & $0.894(2)$ & $0.956(1)$ & $0.875(1)$ & $0.061(8)$ \\
\hline $\mathrm{H}(221)$ & $4 a$ & $0.858(4)$ & $1.170(2)$ & $0.770(2)$ & $0.11(1)$ \\
\hline $\mathrm{H}(222)$ & $4 a$ & $0.792(3)$ & $1.218(2)$ & $0.819(2)$ & $0.10(1)$ \\
\hline $\mathrm{H}(231)$ & $4 a$ & $0.623(3)$ & $1.219(2)$ & $0.750(2)$ & $0.09(1)$ \\
\hline $\mathrm{H}(232)$ & $4 a$ & $0.662(3)$ & $1.137(2)$ & $0.732(1)$ & $0.074(9)$ \\
\hline $\mathrm{H}(25)$ & $4 a$ & $0.404(2)$ & $1.137(1)$ & $0.766(1)$ & $0.037(6)$ \\
\hline $\mathrm{H}(261)$ & $4 a$ & $0.558(3)$ & $1.014(1)$ & $0.778(1)$ & $0.064(8)$ \\
\hline $\mathrm{H}(262)$ & $4 a$ & $0.525(2)$ & $1.058(1)$ & $0.717(1)$ & $0.058(7)$ \\
\hline
\end{tabular}

Table 2. Continued.

\begin{tabular}{|c|c|c|c|c|c|}
\hline Atom & Site & $x$ & $y$ & $z$ & $U_{\text {iso }}$ \\
\hline $\mathrm{H}(271)$ & $4 a$ & $0.407(3)$ & $0.948(2)$ & $0.724(2)$ & $0.09(1)$ \\
\hline $\mathrm{H}(272)$ & $4 a$ & $0.326(2)$ & $1.016(1)$ & $0.711(1)$ & $0.053(7)$ \\
\hline $\mathrm{H}(29)$ & $4 a$ & $0.236(3)$ & $1.041(1)$ & $0.873(1)$ & $0.068(8)$ \\
\hline $\mathrm{H}(301)$ & $4 a$ & $0.659(3)$ & $0.733(2)$ & $0.790(2)$ & $0.08(1)$ \\
\hline $\mathrm{H}(302)$ & $4 a$ & $0.764(3)$ & $0.696(2)$ & $0.828(2)$ & $0.11(1)$ \\
\hline $\mathrm{H}(311)$ & $4 a$ & $0.851(5)$ & $0.766(3)$ & $0.741(3)$ & $0.18(2)$ \\
\hline $\mathrm{H}(312)$ & $4 a$ & $0.806(4)$ & $0.834(2)$ & $0.772(2)$ & $0.11(2)$ \\
\hline $\mathrm{H}(313)$ & $4 a$ & $0.905(8)$ & $0.798(4)$ & $0.811(3)$ & $0.27(4)$ \\
\hline $\mathrm{H}(321)$ & $4 a$ & $0.539(3)$ & $0.680(2)$ & $1.106(2)$ & $0.10(1)$ \\
\hline $\mathrm{H}(322)$ & $4 a$ & $0.569(4)$ & $0.611(2)$ & $1.074(2)$ & $0.12(1)$ \\
\hline $\mathrm{H}(323)$ & $4 a$ & $0.473(4)$ & $0.668(2)$ & $1.039(2)$ & $0.11(1)$ \\
\hline $\mathrm{H}(331)$ & $4 a$ & $0.675(3)$ & $0.855(2)$ & $1.148(2)$ & $0.10(1)$ \\
\hline $\mathrm{H}(332)$ & $4 a$ & $0.712(4)$ & $0.774(2)$ & $1.133(2)$ & $0.12(1)$ \\
\hline $\mathrm{H}(333)$ & $4 a$ & $0.776(4)$ & $0.841(2)$ & $1.100(2)$ & $0.11(1)$ \\
\hline $\mathrm{H}(341)$ & $4 a$ & $0.355(4)$ & $0.936(2)$ & $1.098(2)$ & $0.12(1)$ \\
\hline $\mathrm{H}(342)$ & $4 a$ & $0.418(3)$ & $0.975(2)$ & $1.150(2)$ & $0.10(1)$ \\
\hline $\mathrm{H}(343)$ & $4 a$ & $0.461(3)$ & $0.892(2)$ & $1.152(2)$ & $0.11(1)$ \\
\hline $\mathrm{H}(351)$ & $4 a$ & $0.506(3)$ & $1.150(2)$ & $1.015(2)$ & $0.09(1)$ \\
\hline $\mathrm{H}(352)$ & $4 a$ & $0.587(3)$ & $1.189(2)$ & $1.068(1)$ & $0.09(1)$ \\
\hline $\mathrm{H}(361)$ & $4 a$ & $0.346(5)$ & $1.110(3)$ & $1.086(2)$ & $0.15(2)$ \\
\hline $\mathrm{H}(362)$ & $4 a$ & $0.423(5)$ & $1.156(3)$ & $1.138(3)$ & $0.19(2)$ \\
\hline $\mathrm{H}(363)$ & $4 a$ & $0.360(4)$ & $1.202(3)$ & $1.085(2)$ & $0.14(2)$ \\
\hline $\mathrm{H}(371)$ & $4 a$ & $0.760(5)$ & $1.091(3)$ & $1.177(2)$ & $0.16(3)$ \\
\hline $\mathrm{H}(372)$ & $4 a$ & $0.893(4)$ & $1.114(2)$ & $1.181(2)$ & $0.11(1)$ \\
\hline $\mathrm{H}(373)$ & $4 a$ & $0.861(4)$ & $1.035(2)$ & $1.152(2)$ & $0.15(2)$ \\
\hline $\mathrm{H}(381)$ & $4 a$ & $1.129(4)$ & $1.173(2)$ & $0.980(2)$ & $0.12(2)$ \\
\hline $\mathrm{H}(382)$ & $4 a$ & $1.070(4)$ & $1.158(2)$ & $0.914(2)$ & $0.14(2)$ \\
\hline $\mathrm{H}(383)$ & $4 a$ & $1.102(4)$ & $1.091(2)$ & $0.965(2)$ & $0.12(2)$ \\
\hline $\mathrm{H}(391)$ & $4 a$ & $0.587(3)$ & $1.234(2)$ & $0.873(2)$ & $0.09(1)$ \\
\hline $\mathrm{H}(392)$ & $4 a$ & $0.463(3)$ & $1.239(2)$ & $0.836(2)$ & $0.09(1)$ \\
\hline $\mathrm{H}(393)$ & $4 a$ & $0.476(4)$ & $1.187(2)$ & $0.897(2)$ & $0.12(1)$ \\
\hline $\mathrm{H}(401)$ & $4 a$ & $0.118(3)$ & $0.939(2)$ & $0.826(2)$ & $0.11(1)$ \\
\hline $\mathrm{H}(402)$ & $4 a$ & $0.129(3)$ & $0.962(2)$ & $0.752(1)$ & $0.09(1)$ \\
\hline $\mathrm{H}(411)$ & $4 a$ & $0.249(4)$ & $0.839(2)$ & $0.798(2)$ & $0.13(2)$ \\
\hline $\mathrm{H}(412)$ & $4 a$ & $0.259(4)$ & $0.865(2)$ & $0.724(2)$ & $0.11(1)$ \\
\hline $\mathrm{H}(413)$ & $4 a$ & $0.124(4)$ & $0.833(2)$ & $0.759(2)$ & $0.11(1)$ \\
\hline $\mathrm{H}(421)$ & $4 a$ & $0.087(4)$ & $1.072(2)$ & $0.793(2)$ & $0.11(1)$ \\
\hline $\mathrm{H}(422)$ & $4 a$ & $0.196(3)$ & $1.104(2)$ & $0.750(2)$ & $0.09(1)$ \\
\hline $\mathrm{H}(423)$ & $4 a$ & $0.171(3)$ & $1.140(2)$ & $0.817(1)$ & $0.07(1)$ \\
\hline $\mathrm{H}(13)$ & $4 a$ & $0.760(2)$ & $1.031(1)$ & $1.052(1)$ & $0.048(6)$ \\
\hline $\mathrm{H}(4)$ & $4 a$ & $0.688(3)$ & $0.910(2)$ & $0.966(2)$ & $0.09(1)$ \\
\hline $\mathrm{H}(10)$ & $4 a$ & $0.423(3)$ & $0.912(2)$ & $0.830(2)$ & $0.10(1)$ \\
\hline
\end{tabular}

Table 3. Atomic coordinates and displacement parameters (in $\AA^{2}$ ).

\begin{tabular}{|c|c|c|c|c|c|c|c|c|c|c|}
\hline Atom & Site & $x$ & $y$ & $z$ & $U_{11}$ & $U_{22}$ & $U_{33}$ & $U_{12}$ & $U_{13}$ & $U_{23}$ \\
\hline $\mathrm{C}(01)$ & $4 a$ & $0.6114(3)$ & $0.8498(2)$ & $0.8562(1)$ & $0.069(2)$ & $0.061(2)$ & $0.051(2)$ & $0.001(2)$ & $-0.008(2)$ & $-0.006(1)$ \\
\hline $\mathrm{C}(02)$ & $4 a$ & $0.6769(3)$ & $0.7812(1)$ & $0.8739(1)$ & $0.061(2)$ & $0.053(2)$ & $0.056(2)$ & $0.008(1)$ & $-0.012(1)$ & $-0.012(1)$ \\
\hline $\mathrm{C}(03)$ & $4 a$ & $0.5782(3)$ & $0.7382(1)$ & $0.9098(1)$ & $0.061(2)$ & $0.050(2)$ & $0.069(2)$ & $0.001(1)$ & $-0.011(2)$ & $-0.011(1)$ \\
\hline $\mathrm{C}(04)$ & $4 a$ & $0.6108(4)$ & $0.6617(2)$ & $0.9239(2)$ & $0.086(2)$ & $0.053(2)$ & $0.086(2)$ & $0.001(2)$ & $-0.002(2)$ & $-0.013(2)$ \\
\hline $\mathrm{C}(05)$ & $4 a$ & $0.7063(4)$ & $0.6549(2)$ & $0.9762(2)$ & $0.089(3)$ & $0.056(2)$ & $0.085(2)$ & $0.014(2)$ & $0.002(2)$ & $0.006(2)$ \\
\hline $\mathrm{C}(06)$ & $4 a$ & $0.6640(3)$ & $0.6943(2)$ & $1.0337(2)$ & $0.077(2)$ & $0.063(2)$ & $0.074(2)$ & $0.011(2)$ & $-0.007(2)$ & $0.012(2)$ \\
\hline $\mathrm{C}(08)$ & $4 a$ & $0.6019(3)$ & $0.8212(2)$ & $1.0652(1)$ & $0.063(2)$ & $0.060(2)$ & $0.050(2)$ & $-0.000(1)$ & $-0.001(1)$ & $0.005(1)$ \\
\hline $\mathrm{C}(09)$ & $4 a$ & $0.5819(3)$ & $0.8939(1)$ & $1.0372(1)$ & $0.058(2)$ & $0.058(2)$ & $0.048(1)$ & $-0.006(1)$ & $0.003(1)$ & $-0.003(1)$ \\
\hline$C(10)$ & $4 a$ & $0.5515(3)$ & $0.9514(1)$ & $1.0840(1)$ & $0.072(2)$ & $0.057(2)$ & $0.043(1)$ & $-0.007(2)$ & $0.002(1)$ & $-0.002(1)$ \\
\hline $\mathrm{C}(11)$ & $4 a$ & $0.5277(3)$ & $1.0195(1)$ & $1.0499(1)$ & $0.072(2)$ & $0.068(2)$ & $0.039(1)$ & $0.001(2)$ & $0.009(1)$ & $-0.011(1)$ \\
\hline $\mathrm{C}(12)$ & $4 a$ & $0.5926(3)$ & $1.0843(2)$ & $1.0748(1)$ & $0.096(2)$ & $0.056(2)$ & $0.040(2)$ & $0.005(2)$ & $0.004(2)$ & $-0.006(1)$ \\
\hline $\mathrm{C}(13)$ & $4 a$ & $0.7310(3)$ & $1.0810(2)$ & $1.0529(1)$ & $0.088(2)$ & $0.048(2)$ & $0.043(1)$ & $0.000(2)$ & $-0.006(1)$ & $-0.006(1)$ \\
\hline $\mathrm{C}(14)$ & $4 a$ & $0.8254(4)$ & $1.1213(2)$ & $1.0922(1)$ & $0.102(3)$ & $0.062(2)$ & $0.054(2)$ & $-0.001(2)$ & $-0.019(2)$ & $-0.009(2)$ \\
\hline$C(15)$ & $4 a$ & $0.9522(4)$ & $1.1184(2)$ & $1.0604(1)$ & $0.093(3)$ & $0.069(2)$ & $0.068(2)$ & $-0.009(2)$ & $-0.030(2)$ & $-0.010(2)$ \\
\hline$C(17)$ & $4 a$ & $0.8458(3)$ & $1.1059(1)$ & $0.9566(1)$ & $0.064(2)$ & $0.056(2)$ & $0.054(2)$ & $-0.005(1)$ & $-0.006(1)$ & $-0.006(1)$ \\
\hline $\mathrm{C}(18)$ & $4 a$ & $0.8791(3)$ & $1.0321(2)$ & $0.9401(1)$ & $0.062(2)$ & $0.058(2)$ & $0.068(2)$ & $-0.000(1)$ & $-0.003(2)$ & $-0.005(2)$ \\
\hline$C(19)$ & $4 a$ & $0.8758(3)$ & $1.0071(2)$ & $0.8826(1)$ & $0.058(2)$ & $0.064(2)$ & $0.078(2)$ & $-0.001(2)$ & $0.002(2)$ & $-0.019(2)$ \\
\hline $\mathrm{C}(20)$ & $4 a$ & $0.8359(2)$ & $1.0507(2)$ & $0.8313(1)$ & $0.047(2)$ & $0.080(2)$ & $0.064(2)$ & $-0.008(2)$ & $0.000(1)$ & $-0.020(2)$ \\
\hline $\mathrm{C}(21)$ & $4 a$ & $0.7735(3)$ & $1.1198(1)$ & $0.8487(1)$ & $0.061(2)$ & $0.064(2)$ & $0.047(1)$ & $-0.014(1)$ & $-0.000(1)$ & $-0.005(1)$ \\
\hline $\mathrm{C}(22)$ & $4 a$ & $0.7828(4)$ & $1.1761(2)$ & $0.7989(2)$ & $0.091(3)$ & $0.084(3)$ & $0.054(2)$ & $-0.029(2)$ & $-0.003(2)$ & $0.007(2)$ \\
\hline
\end{tabular}


Table 3. Continued.

\begin{tabular}{|c|c|c|c|c|c|c|c|c|c|c|}
\hline Atom & Site & $x$ & $y$ & $z$ & $U_{11}$ & $U_{22}$ & $U_{33}$ & $U_{12}$ & $U_{13}$ & $U_{23}$ \\
\hline $\mathrm{C}(23)$ & $4 a$ & $0.6550(3)$ & $1.1738(2)$ & $0.7665(1)$ & $0.089(2)$ & $0.070(2)$ & $0.055(2)$ & $-0.017(2)$ & $-0.005(2)$ & $0.012(2)$ \\
\hline$C(24)$ & $4 a$ & $0.5640(3)$ & $1.1492(1)$ & $0.8171(1)$ & $0.072(2)$ & $0.052(2)$ & $0.050(2)$ & $0.008(2)$ & $-0.003(1)$ & $0.005(1)$ \\
\hline$C(25)$ & $4 a$ & $0.4547(3)$ & $1.1057(1)$ & $0.7925(1)$ & $0.056(2)$ & $0.061(2)$ & $0.043(1)$ & $0.007(2)$ & $0.002(1)$ & $0.012(1)$ \\
\hline$C(26)$ & $4 a$ & $0.4911(3)$ & $1.0411(2)$ & $0.7560(1)$ & $0.060(2)$ & $0.075(2)$ & $0.045(2)$ & $-0.002(2)$ & $0.004(1)$ & $-0.006(1)$ \\
\hline$C(27)$ & $4 a$ & $0.3767(3)$ & $0.9948(2)$ & $0.7422(1)$ & $0.058(2)$ & $0.080(2)$ & $0.048(2)$ & $-0.005(2)$ & $-0.005(1)$ & $0.001(2)$ \\
\hline $\mathrm{C}(28)$ & $4 a$ & $0.2964(3)$ & $0.9808(2)$ & $0.7997(1)$ & $0.058(2)$ & $0.067(2)$ & $0.049(1)$ & $0.004(1)$ & $-0.004(1)$ & $0.012(1)$ \\
\hline $\mathrm{C}(29)$ & $4 a$ & $0.2637(3)$ & $1.0498(2)$ & $0.8302(1)$ & $0.058(2)$ & $0.074(2)$ & $0.060(2)$ & $0.005(2)$ & $0.012(1)$ & $0.016(2)$ \\
\hline $\mathrm{C}(30)$ & $4 a$ & $0.7267(4)$ & $0.7450(2)$ & $0.8154(2)$ & $0.089(3)$ & $0.076(2)$ & $0.069(2)$ & $0.015(2)$ & $-0.006(2)$ & $-0.022(2)$ \\
\hline $\mathrm{C}(31)$ & $4 a$ & $0.8265(6)$ & $0.7866(3)$ & $0.7819(2)$ & $0.131(4)$ & $0.123(4)$ & $0.074(3)$ & $-0.007(3)$ & $0.026(3)$ & $-0.009(3)$ \\
\hline $\mathrm{C}(32)$ & $4 a$ & $0.5510(5)$ & $0.6614(2)$ & $1.0651(2)$ & $0.115(4)$ & $0.074(3)$ & $0.092(3)$ & $-0.006(2)$ & $0.017(3)$ & $0.023(2)$ \\
\hline $\mathrm{C}(33)$ & $4 a$ & $0.7003(5)$ & $0.8218(2)$ & $1.1172(2)$ & $0.103(3)$ & $0.085(3)$ & $0.061(2)$ & $0.010(2)$ & $-0.019(2)$ & $0.000(2)$ \\
\hline $\mathrm{C}(34)$ & $4 a$ & $0.4368(4)$ & $0.9370(2)$ & $1.1253(2)$ & $0.103(3)$ & $0.076(2)$ & $0.063(2)$ & $-0.009(2)$ & $0.025(2)$ & $-0.011(2)$ \\
\hline$C(35)$ & $4 a$ & $0.5237(4)$ & $1.1522(2)$ & $1.0595(2)$ & $0.109(3)$ & $0.062(2)$ & $0.076(2)$ & $0.021(2)$ & $0.016(2)$ & $-0.005(2)$ \\
\hline$C(36)$ & $4 a$ & $0.3946(6)$ & $1.1579(3)$ & $1.0909(3)$ & $0.145(4)$ & $0.106(4)$ & $0.119(4)$ & $0.055(4)$ & $0.052(3)$ & $0.007(3)$ \\
\hline $\mathrm{C}(37)$ & $4 a$ & $0.8333(7)$ & $1.0902(3)$ & $1.1583(2)$ & $0.149(5)$ & $0.130(4)$ & $0.058(2)$ & $-0.015(4)$ & $-0.036(3)$ & $0.002(2)$ \\
\hline $\mathrm{C}(38)$ & $4 a$ & $1.0777(4)$ & $1.1417(3)$ & $0.9623(2)$ & $0.079(3)$ & $0.117(4)$ & $0.101(3)$ & $-0.029(3)$ & $-0.009(2)$ & $-0.017(3)$ \\
\hline $\mathrm{C}(39)$ & $4 a$ & $0.5175(5)$ & $1.2091(2)$ & $0.8576(2)$ & $0.107(3)$ & $0.063(2)$ & $0.086(3)$ & $0.017(2)$ & $-0.008(3)$ & $-0.014(2)$ \\
\hline $\mathrm{C}(40)$ & $4 a$ & $0.1770(3)$ & $0.9374(2)$ & $0.7847(2)$ & $0.065(2)$ & $0.099(3)$ & $0.076(2)$ & $-0.011(2)$ & $-0.007(2)$ & $0.027(2)$ \\
\hline $\mathrm{C}(41)$ & $4 a$ & $0.2048(5)$ & $0.8629(2)$ & $0.7658(2)$ & $0.104(3)$ & $0.093(3)$ & $0.092(3)$ & $-0.034(3)$ & $-0.012(3)$ & $0.002(2)$ \\
\hline $\mathrm{C}(42)$ & $4 a$ & $0.1721(4)$ & $1.0965(2)$ & $0.7951(2)$ & $0.059(2)$ & $0.090(3)$ & $0.100(3)$ & $0.017(2)$ & $0.001(2)$ & $0.025(2)$ \\
\hline $\mathrm{O}(01)$ & $4 a$ & $0.6414(2)$ & $0.90506(9)$ & $0.88525(8)$ & $0.079(1)$ & $0.055(1)$ & $0.059(1)$ & $0.000(1)$ & $-0.014(1)$ & $-0.0038(9)$ \\
\hline $\mathrm{O}(02)$ & $4 a$ & $0.5301(2)$ & $0.8474(1)$ & $0.81358(9)$ & $0.097(2)$ & $0.073(1)$ & $0.073(1)$ & $0.017(1)$ & $-0.036(1)$ & $-0.013(1)$ \\
\hline $\mathrm{O}(03)$ & $4 a$ & $0.5452(2)$ & $0.77336(9)$ & $0.96674(8)$ & $0.056(1)$ & $0.055(1)$ & $0.061(1)$ & $0.0029(9)$ & $-0.0074(9)$ & $-0.0047(8)$ \\
\hline $\mathrm{O}(04)$ & $4 a$ & $0.6941(2)$ & $0.9170(1)$ & $1.00573(9)$ & $0.076(1)$ & $0.065(1)$ & $0.051(1)$ & $-0.014(1)$ & $0.009(1)$ & $-0.0081(9)$ \\
\hline $\mathrm{O}(05)$ & $4 a$ & $0.4607(2)$ & $1.0216(1)$ & $1.00390(8)$ & $0.085(1)$ & $0.082(1)$ & $0.048(1)$ & $0.009(1)$ & $-0.006(1)$ & $-0.0075(9)$ \\
\hline $\mathrm{O}(06)$ & $4 a$ & $0.7281(2)$ & $1.10668(9)$ & $0.98957(7)$ & $0.071(1)$ & $0.058(1)$ & $0.0427(9)$ & $0.001(1)$ & $-0.0051(9)$ & $-0.0049(8)$ \\
\hline $\mathrm{O}(07)$ & $4 a$ & $0.8259(2)$ & $1.14811(9)$ & $0.90348(8)$ & $0.076(1)$ & $0.058(1)$ & $0.048(1)$ & $-0.013(1)$ & $-0.0066(9)$ & $-0.0024(9)$ \\
\hline $\mathrm{O}(08)$ & $4 a$ & $0.6423(2)$ & $1.10306(9)$ & $0.85554(7)$ & $0.054(1)$ & $0.055(1)$ & $0.0478(9)$ & $-0.0019(9)$ & $-0.0013(8)$ & $0.0036(8)$ \\
\hline $\mathrm{O}(09)$ & $4 a$ & $0.3796(2)$ & $1.08681(9)$ & $0.84559(7)$ & $0.067(1)$ & $0.064(1)$ & $0.0441(9)$ & $0.012(1)$ & $0.0077(9)$ & $0.0029(8)$ \\
\hline $\mathrm{O}(10)$ & $4 a$ & $0.3694(2)$ & $0.9455(1)$ & $0.84572(9)$ & $0.074(1)$ & $0.066(1)$ & $0.055(1)$ & $0.012(1)$ & $-0.010(1)$ & $0.0057(9)$ \\
\hline $\mathrm{O}(11)$ & $4 a$ & $0.8447(2)$ & $1.0331(1)$ & $0.7768(1)$ & $0.084(2)$ & $0.127(2)$ & $0.067(1)$ & $0.013(1)$ & $0.004(1)$ & $-0.035(1)$ \\
\hline $\mathrm{Na}(1)$ & $4 a$ & $0.5241(1)$ & $1.00625(5)$ & $0.90272(4)$ & $0.0843(7)$ & $0.0585(6)$ & $0.0445(5)$ & $0.0056(6)$ & $-0.0072(5)$ & $0.0011(5)$ \\
\hline
\end{tabular}

\section{References}

1. Japan Kokai Tokkyo Koho, Jp 57085390 A2, May 28th, 1982.

2. Flack, H. D.; Bernardinelli, G.: Absolute structure and absolute configuration. Acta Crystallogr. A55 (1999) 908-915.

3. Hamilton, W. C.: Significance Tests on the Crystallographic R Factors. Acta Crystallogr. 18 (1965) 502-510.

4. Miyazaki, Y.; Shibuya, M.; Sugawara, H.; Kawaguchi, O.; Hirsoe, C.: Salinomycin, a new polyether antibiotic. J. Antibiotics 27 (1974) 814-821.

5. Miyazaki, Y; Shibata, A.; Tsuda, K.; Kinashi, H.; Otake, N.: Studies on the ionophorous antibiotics. Isolation, characterisation and structure of SY-1. Agric. Biol. Chem. 42 (1978) 2129-2132.

6. Kinashi, H.; Otake, N.; Yonehara, H.; Sato, S.; Saito, Y.: Studies on the Ionophorous Antibiotics. I. The Crystal and Molecular Structure of Salinomycin $p$-Iodophenacyl Ester. Acta Crystallogr. B31 (1975) 24112415.

7. Paulus, E. F.; Kurz, M.; Matter, H.; Vértesy, L.: Solid-State and Solution Structure of the Salinomycin-Sodium Complex: Stabilization of Different Conformers for an Ionophore in Different Environments. J. Am. Chem. Soc. 120 (1998) 8209-8221.
8. Paulus, E. F.; Vértesy, L.: The Antibiotic Salinomycin and another three Derivatives. Lecture MS2.6-6, 17th European Crystallographic Meeting, Lisboa 1997; Poster, Computing School on Practical Aspects of Charge Density Determination, Berlin 1997.

9. Paulus, E. F.; Vértesy, L.: Crystal structure of the antibiotic SY-1 (20-deoxysalinomycin): sodium 2-(6-[2-(5-ethyl-5-hydroxy-6-methyl-tetrahydropyran-2-yl)-2,10,12-trimethyl-1,6,8-trioxa-dispiro[4.1.5.3]-pentadec13-en-9-yl]-2-hydroxy-1,3-dimethyl-4-oxo-heptyl-5-methyl-tetrahydropyran-2-yl)-butyrate, $\mathrm{C}_{42} \mathrm{H}_{69} \mathrm{NaO}_{10} \cdot 0.69 \mathrm{CH}_{3} \mathrm{OH}$. Z. Kristallogr. NCS 218 (2003) 575-577.

10. Sheldrick, G. M.: SHELXS-97. Program for the Solution of Crystal Structures. University of Göttingen, Germany 1997.

11. Sheldrick, G. M.: SHELXL-97. Program for the Refinement of Crystal Structures. University of Göttingen, Germany 1997.

12. Sheldrick, G. M.: SHELXTL-PLUS, Release 5.10. An Integrated System for Solving, Refining and Displaying Crystal Structures from Diffraction Data. Bruker Analytical Systems, Madison, Wisconsin USA 1997. 